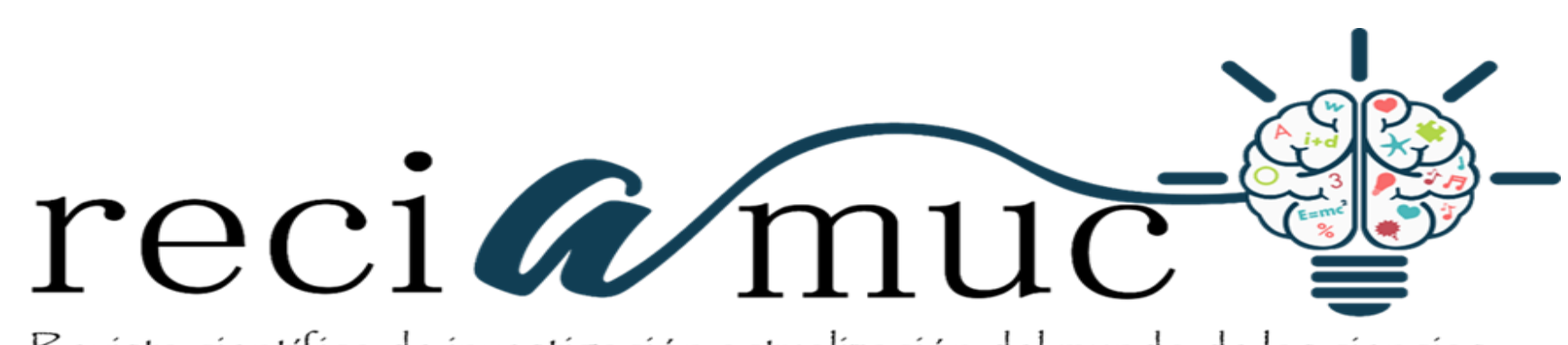

Revista científica de investigación actualización del mundo de las ciencias

Giannella Lissette Ramírez López a; Nancy del Carmen López García ${ }^{\text {b }}$; Cinthya Clara Moreira Simbaña ${ }^{c}$; Jenny Marisela Briones Fajardo ${ }^{d}$

Control del dolor postquirúrgico

Post-surgical pain control

Revista Científica de Investigación actualización del mundo de las Ciencias. Vol. 3 núm., 2, abril, ISSN: 2588-0748, 2018, pp. 712-724

DOI: $\underline{10.26820 / \text { reciamuc/3.(2).abril.2019.712-724 }}$

URL: http://reciamuc.com/index.php/RECIAMUC/article/view/363

Código UNESCO: 3205 Medicina Interna

Tipo de Investigación: Artículo de Revisión

(C) RECIAMUC; Editorial Saberes del Conocimiento, 2019

Recibido: $15 / 01 / 2019$

Aceptado: 07/02/2019

Publicado: 01/04/2019

Correspondencia: giannelita021292@gmail.com

a. Médico; Investigador Independiente; Guayaquil, Ecuador;giannelita021292@gmail.com

b. Magister en Salud Pública, Diplomado Superior de Cuarto Nivel en Desarrollo Local y Salud, Licencia en Enfermería; Investigador Independiente; Guayaquil, Ecuador;nancylopez-g@ hotmail.com

c. Médico; Investigador Independiente; Guayaquil, Ecuador;cinthya-moreira-373@hotmail.com

d. Médico; Investigador Independiente; Guayaquil, Ecuador;jeimmy_b@ hotmail.com 
Control del dolor postquirúrgico

Vol. 3, núm. 2., (2019)

Giannella Lissette Ramírez López; Nancy del Carmen López García; Cinthya Clara Moreira

Simbaña; Jenny Marisela Briones Fajardo

\section{RESUMEN}

El dolor postoperatorio es un síntoma de la cirugía que se presenta persistentemente hasta en un $60 \%$ de los pacientes en todo el mundo, que de no ser controlado adecuadamente puede aumentar el riesgo de complicaciones, morbilidad y mortalidad. El objetivo fundamental de la presente investigación es plasmar el control del dolor postquirúrgico, desde la perspectiva de las innovaciones presentadas en la actualidad: 1 . Tratamiento farmacológico del dolor postquirúrgico. 2. Nuevas vías de administración de opioides. 3. Recomendaciones y guías para el control del dolor postquirúrgico. El diseño de investigación que se llevó a cabo es de tipo documental o bibliográfico. En la actualidad se siguen usando los mismos fármacos para el control del dolor postquirúrgico, no obstante, las innovaciones se presentan en la vía de administración. Asimismo, las guías y recomendaciones internacionales sirven como base a cada región del planeta, país y/o centro hospitalario para la unificación de criterios y elaboración de protocolos que garanticen el bienestar integral del paciente operado. En conclusión, las innovaciones presentadas hasta la actualidad enmarcan el camino hacia la garantía de la reducción de las complicaciones asociadas a los tratamientos del dolor postquirúrgico y de la recuperación plena y satisfactoria del paciente.

Palabras claves: Control; Dolor; Postquirúrgico; Tratamiento; Innovaciones. 


\title{
Control del dolor postquirúrgico
}

Vol. 3, núm. 2., (2019)

Giannella Lissette Ramírez López; Nancy del Carmen López García; Cinthya Clara Moreira Simbaña; Jenny Marisela Briones Fajardo

\begin{abstract}
Postoperative pain is a symptom of surgery that persistently occurs in up to $60 \%$ of patients worldwide, which if not properly controlled can increase the risk of complications, morbidity and mortality. The main objective of this research is to capture the control of post-surgical pain, from the perspective of the innovations presented today: 1. Pharmacological treatment of post-surgical pain. 2. New routes of opioid administration. 3. Recommendations and guidelines for post-surgical pain control. The research design that was carried out is documentary or bibliographic. Currently, the same drugs are still used for post-surgical pain control, however, innovations are presented in the route of administration. Likewise, international guides and recommendations serve as the basis for each region of the planet, country and / or hospital center for the unification of criteria and the elaboration of protocols that guarantee the integral well-being of the operated patient. In conclusion, the innovations presented to date frame the way towards guaranteeing the reduction of complications associated with post-surgical pain treatments and the full and satisfactory recovery of the patient.
\end{abstract}

Key words: Control; Pain; Post-surgical; Treatment; Innovations. 


\section{Control del dolor postquirúrgico}

Vol. 3, núm. 2., (2019)

Giannella Lissette Ramírez López; Nancy del Carmen López García; Cinthya Clara Moreira

Simbaña; Jenny Marisela Briones Fajardo

\section{Introducción.}

En la actualidad, más de 300 millones de cirugías se realizan durante todo el año a nivel mundial, siendo el dolor su síntoma más frecuente. De los pacientes intervenidos quirúrgicamente, se estima que entre el 25 y el $60 \%$ sufre dolor moderado o severo. La presencia del dolor, así como la intensidad o el periodo de tiempo que se presente, varía de un paciente a otro. Un $60 \%$ aproximadamente de los pacientes que presentaron dolor postoperatorio, sufre este dolor persistentemente. No obstante, existe forma de prevenirlo o controlarlo a los fines de minimizar las consecuencias negativas que conlleva. (Sociedad Española del Dolor, 2017)

Para Aguilar, Montes, Benito, Caba, \& Margarit (2018) se refiere al dolor agudo postoperatorio como el "conjunto de percepciones sensoriales, emocionales y mentales desagradables, asociadas a respuestas autonómicas, psicológicas y conductuales que han sido precipitadas como consecuencia del acto quirúrgico”. (p. 71)

En cuanto a su prevalencia, Gant y cols., citados por Esteve, Sansaloni, Verd, Ribera, \& Mora, (2017) realizaron una encuesta en 300 pacientes donde obtuvieron resultados muy similares a algunos estudios realizados los últimos 20 años, lo que hace concluir que la prevalencia del dolor agudo postoperatorio se ha modificado mínimamente durante este período de tiempo, ya que se mantiene entre un 45-49\%, para la categoría de dolor intenso entre un 21-23\% y para el dolor máximo de un 8 a $18 \%$. (p. 133)

Estas estadísticas colocan al dolor en un lugar importante entre los aspectos perioperatorios, que debe ser considerado desde antes de la cirugía, en el momento de la misma, y posteriormente. 


\section{Control del dolor postquirúrgico}

Vol. 3, núm. 2., (2019)

Giannella Lissette Ramírez López; Nancy del Carmen López García; Cinthya Clara Moreira Simbaña; Jenny Marisela Briones Fajardo

Este síntoma de la cirugía debe ser tratado adecuadamente ya que va a influir proporcionalmente en la recuperación del paciente y el éxito total del procedimiento quirúrgico.

Existen factores que pueden estar asociados a la cantidad de dolor y a cómo manejarlo, entre los tenemos el tipo de cirugía y técnicas utilizadas. También influyen el grado de complejidad que presente la cirugía y el tiempo que tarde en realizarse. En todo caso, el control del dolor es fundamental para la recuperación del paciente, de ello depende que pueda levantarse y comenzar a moverse, y ello repercute en la disminución de riesgo de coágulos sanguíneos en los miembros inferiores o los pulmones, de infecciones pulmonares y urinarias, reduce la estancia hospitalaria y reduce los costos. (Biblioteca Nacional de Medicina de los Estados Unidos, 2017)

El tratamiento estándar para el control del dolor después de una operación se realiza por medio de analgésicos, los cuales tienen la función de reducir o aliviar el dolor del paciente operado.

“Los analgésicos recetados más potentes son los opioides. Son muy efectivos, pero quienes los toman tienen riesgos de sufrir adicción o sobredosis". (Biblioteca Nacional de Medicina de los Estados Unidos, 2019)

Humble et al., citado en Quinde Cobos (2017) refiere que, si no se controla bien el dolor postoperatorio, esto contribuye con: "una peor experiencia de la enfermedad para el paciente, menor satisfacción, deambulación tardía, aumento de incidencia de complicaciones cardíacas y pulmonares, y aumento de morbilidad y mortalidad”. (p. 2) 


\section{Control del dolor postquirúrgico}

Vol. 3, núm. 2., (2019)

Giannella Lissette Ramírez López; Nancy del Carmen López García; Cinthya Clara Moreira Simbaña; Jenny Marisela Briones Fajardo

En la actualidad se presentan diversos retos en el campo del tratamiento del dolor postoperatorio que requieren, imperiosamente, de novedosos puntos de vista, es necesaria una nueva proyección en la forma de atención a este problema y exige nuevas opciones en la terapia.

El objetivo principal del presente estudio consiste en revisar y plasmar el tema del control del dolor postquirúrgico, desde la perspectiva de las innovaciones presentadas en la actualidad: 1 . Tratamiento farmacológico del dolor postquirúrgico. 2. Nuevas vías de administración de opioides. 3. Recomendaciones y guías para el control del dolor postquirúrgico (unidad de criterios y protocolos basados en evidencia).

\section{Materiales y Métodos}

Para el desarrollo de la presente investigación se usaron computadores personales con conexión a internet, con el propósito de exponer acerca del control del dolor postquirúrgico, en virtud de lo cual, el mismo se enmarca en un diseño de investigación documental o bibliográfica.

Esta investigación se limita a la búsqueda y revisión sistemática de material bibliográfico que, por una parte, es accesible mediante el uso de diversas bases de datos, entre las que figuraron: MedlinePlus, PubMed, Biblioteca Virtual de la Salud (BVS), SciELO, Dialnet y ELSEVIER, entre otras; mediante las cuales se sintetizó la mejor evidencia disponible.

Se realizó una búsqueda aleatoria y consecutiva, usando las expresiones "control del dolor postquirúrgico", "tratamiento del dolor postoperatorio"; "dolor postquirúrgico" y "guías para el tratamiento del dolor postoperatorio", lo que aproximadamente resultó en más de un centenar de miles de registros bibliográficos que luego se fueron filtrando bajo los criterios de idioma español, 


\section{Control del dolor postquirúrgico}

Vol. 3, núm. 2., (2019)

Giannella Lissette Ramírez López; Nancy del Carmen López García; Cinthya Clara Moreira Simbaña; Jenny Marisela Briones Fajardo

relevancia, correlación temática y fecha de publicación en los últimos cinco años, sin descartar por tipo de material bibliográfico.

Por último, se le da la correspondiente lectura crítica y análisis de toda esa evidencia científica, lo que resultó consecutiva y consensuadamente en el contenido del presente trabajo.

\section{Resultados}

Tratamiento farmacológico para el control del dolor postquirúrgico.

El tratamiento del dolor posquirúrgico, en líneas generales, se busca controlar farmacológicamente por medio de analgésicos. Para su administración se debe considerar como aspectos relevantes: el tipo de analgésico, la vía de administración y la dosis. Existe una amplia gama de analgésicos para el control del dolor postquirúrgico, entre los que se encuentran los opioides, que son unos de los más poderosos analgésicos para disminuir la percepción del dolor, “incluyen fentanilo, hidromorfona, morfina, oxicodona, oximorfona y tramadol”. También son usados los anestésicos locales que causan pérdida de la sensibilidad, tales como la lidocaína y la bupivacaína. Asimismo, son administrados antiinflamatorios no esteroideos (AINE), como en el caso del ibuprofeno (Advil, Motrin IB y otros) o naproxeno (Aleve, Anaprox y otros), que actúan reduciendo las inflamaciones que colaboran con la presencia del dolor. Existen otros analgésicos no opioides como el paracetamol/acetaminofeno (Tylenol y otros), entre otros. (Mayo Clinic, 2018)

Para Jiménez (2018) "la analgesia postoperatoria con Morfina espinal, es el estándar de oro para el dolor agudo". Para su administración se deben usar dosis bajas a los fines de evitar efectos 


\section{Control del dolor postquirúrgico}

Vol. 3, núm. 2., (2019)

Giannella Lissette Ramírez López; Nancy del Carmen López García; Cinthya Clara Moreira Simbaña; Jenny Marisela Briones Fajardo

secundarios de gravedad. Se recomienda su uso bajo un esquema multimodal y/o dosis de rescate. (p. 43,44)

El Ketorolato es un analgésico usado con preferencia en el postoperatorio de las cirugías de fracturas. Bermejo et al. (2019) llevó a cabo un estudio en una población de 101 individuos, mayores de 12 años de ambos sexos, con un diagnóstico de fracturas de extremidades, ingresados al servicio de traumatología de un hospital de segundo nivel de la provincia de Cañar, Ecuador, durante el periodo de enero a julio del año 2017, en el cual encontró que en el $93 \%$ de las prescripciones para tratar el dolor postquirúrgico en estos casos se usó ketorolaco, como terapia única o asociado a otro fármaco. Asimismo, los autores citan a Sánchez y Moreno, los que obtuvieron en su trabajo cifras similares, es decir, estos obtuvieron un $78.7 \%$ y $94.8 \%$, respectivamente, de prescripción de este fármaco. (p. 197)

Nuevas vías de administración de opioides.

Las innovaciones analgésicas más resaltantes están basadas en el uso de viejos fármacos, pero con nuevos dispositivos o vías de administración. Novedosos dispositivos de administración de opioides no invasivos o "needel-free", se han desarrollado con la finalidad de eliminar las complicaciones o desventajas de la analgesia intravenosa controlada por el paciente de morfina. El sufentanilo sublingual se encuentra aprobado en la Unión Europea y pendiente por la aprobación de la FDA (Food and Drug Administration). Se trata de un dispositivo preprogramado que “administra nanotabletas sublinguales de 15 microgramos de sufentanil a demanda del paciente, con un tiempo de seguridad de 20 minutos, durante un periodo de 72 h". El sufentanilo sublingual presenta un índice terapéutico elevado, además de una elevada biodisponibilidad y un tiempo 


\section{Control del dolor postquirúrgico}

Vol. 3, núm. 2., (2019)

Giannella Lissette Ramírez López; Nancy del Carmen López García; Cinthya Clara Moreira Simbaña; Jenny Marisela Briones Fajardo

rápido de equilibrio entre el plasma y el sistema nervioso central. Además de esto, carece de metabolitos activos y la edad del paciente, su índice corporal o la insuficiencia renal o hepática, son factores que no altera su metabolismo. Por último, el fentanilo transdérmico reinsertado en el mercado, tiene un rápido inicio de acción y su índice terapéutico es mayor que la morfina (Esteve et al., 2017, p. 137, 138)

Recomendaciones y guías para el control del dolor postquirúrgico (unidad de criterios y protocolos basados en evidencia).

A nivel internacional existen recomendaciones y guías para el control o tratamiento del dolor postoperatorio que unifican criterios en base a la evidencia con el propósito de garantizar el mejor manejo del dolor y el mayor beneficio para el paciente operado.

En la actualidad, una de las más novedosas y usadas Guías en todo el mundo para el manejo del dolor postoperatorio es la guía de práctica clínica publicada por la Asociación Americana del Dolor (American Pain Society- APS), en febrero del 2016 y ofrece 32 recomendaciones basadas en la evidencia para el manejo del dolor postoperatorio en niños y adultos. Está elaborada con la colaboración de un equipo multidisciplinario conformado por la Sociedad Americana de Anestesiología (American Society of Anesthesiologists), y dicho el documento posteriormente fue aprobado por la Sociedad Americana de Anestesia Regional y Tratamiento del Dolor (American Society of Regional Anesthesia and Pain Management). Esta guía es una valiosa herramienta en la elaboración de planes específicos de control del dolor postoperatorio y la base para desarrollar nuevas investigaciones y llenar las lagunas presentes en estos procedimientos. 


\section{Control del dolor postquirúrgico}

Vol. 3, núm. 2., (2019)

Giannella Lissette Ramírez López; Nancy del Carmen López García; Cinthya Clara Moreira Simbaña; Jenny Marisela Briones Fajardo

Acerca de la guía es importante destacar entre sus recomendaciones más importantes, "el uso de la analgesia multimodal, la analgesia regional y epidural en procedimientos específicos, las mínimas dosis de opioides y la preferencia de la vía oral frente a la intravenosa, y la modalidad de analgesia controlada por el paciente". (Esteve et al., 2017, p. 135)

Alcántara \& González (2016) refieren en su estudio las cuatro recomendaciones basadas en evidencia de alta calidad de la guía de práctica clínica para el manejo de dolor postquirúrgico de la APS: 1. Analgesia multimodal, 2. Uso de paracetamol y/o antiinflamatorios no esteroideos como parte de la analgesia multimodal..., ...3. Consideración de técnicas de anestesia regional periférica específicas..., ...4. Ofrecer la analgesia neuroaxial para los principales procedimientos torácicos y abdominales...”. (p. 164)

La terapia multimodal se define el método que permite el uso de varios fármacos y diversas técnicas, de forma combinadas, pero con distintos mecanismos de acción en el sistema nervioso central y/o periférico. El uso de opioides presenta algunas desventajas y el riesgo de estas aumenta con su uso a largo plazo, mediante la terapia multimodal puede reducirse estos riesgos al combinarlo con dosis de fármacos no opioides y con terapia no farmacológica.

El manejo del dolor postoperatorio debe ser manejado tomando en cuenta la educación preoperatoria, el planeamiento del dolor perioperatorio y el uso de varias modalidades farmacológicas y no farmacológicas, con la finalidad de elaborar políticas y procedimientos en cada institución para brindar la garantía del bienestar del paciente durante su permanencia y egreso del centro de salud, incluso fuera de este. Uno de los puntos resaltantes de la guía APS es acerca de la estructura organizacional, políticas y procedimientos, ante lo cual recomiendan que en 


\section{Control del dolor postquirúrgico}

Vol. 3, núm. 2., (2019)

Giannella Lissette Ramírez López; Nancy del Carmen López García; Cinthya Clara Moreira Simbaña; Jenny Marisela Briones Fajardo

aquellos "centros donde se realicen procedimientos quirúrgicos y analgesia neuraxial exista una estructura organizacional que evalúe la implementación y desarrollo de políticas y prácticas para asegurar el cumplimiento de protocolos seguros y basados en evidencia”. (Quinde Cobos, 2017, p. 9)

Con relación a lo anterior, en la actualidad los centros hospitalarios en todo el mundo han dado mayor importancia a las Unidades de Dolor Agudo (UDA) y los programas de gestión del dolor postoperatorio.

\section{Conclusión.}

Los aspectos innovadores para el control del dolor postquirúrgico en la actualidad, representan el nuevo camino hacia la garantía de la reducción de los costos hospitalarios (que implica la estancia del paciente y los gastos que de ello se derivan), minimización de las complicaciones y efectos secundarios de la acción directa de los fármacos usados en el tratamiento, la reducción de la inamovilidad y de las complicaciones derivadas y en general, de la recuperación plena y satisfactoria del paciente.

El tratamiento farmacológico es uno de los aspectos que más se ha mantenido en el tiempo, presentándose las innovaciones más en la vía de administración de los mismos.

Las guías y recomendaciones internacionales buscan evitar la falta de manejo organizado y multidisciplinario y sirven como base para el desarrollo de los protocolos a usar para el manejo del dolor postquirúrgico de acuerdo a la necesidad particular y nuevas evidencias. 


\section{Control del dolor postquirúrgico}

Vol. 3, núm. 2., (2019)

Giannella Lissette Ramírez López; Nancy del Carmen López García; Cinthya Clara Moreira Simbaña; Jenny Marisela Briones Fajardo

La literatura existente ofrece esquemas heterogéneos acerca del manejo del dolor postquirúrgica, razón por la cual, resulta fundamental que cada centro de salud desarrolle sus propios protocolos con base a las recomendaciones internacionales, guías efectivas y de fácil comprensión y uso, con la finalidad de llegar a un consenso y que todo el equipo de salud relacionado con la cirugía maneje un mismo criterio.

Ocurre en muchos centros hospitalarios y por parte de muchos médicos que las técnicas aplicadas para el control del dolor postquirúrgico están más relacionadas con la forma como el propio cirujano está acostumbrado a manejar este tipo de situación que con la magnitud del dolor que expresa el paciente, y esta es una de las más importantes razones de unificar criterios por medio de guías, así como la formación del paciente y la accesibilidad integral de estos protocolos.

\section{Referencias Bibliográficas}

Aguilar, J. L., Montes, A., Benito, C., Caba, F., \& Margarit, C. (2018). Manejo farmacológico del dolor agudo postoperatorio en España. Datos de la encuesta nacional de la Sociedad Española del Dolor (SED). Rev Soc Esp Dolor, 25(2), 70-85. Recuperado el 04 de agosto de 2019, de https://www.resed.es/Documentos/ArticulosNew/04_OR_Aguilar.pdf

Alcántara, A., \& González, A. (2016). Nueva Guía de la Asociación Americana del Dolor sobre dolor postoperatorio. sobre dolor postoperatorio, 23(3), 164-165. Recuperado el 06 de agosto de 2019, de http://scielo.isciii.es/pdf/dolor/v23n3/08_carta.pdf

Bermejo, D., Álvarez, R., Lliguisupa, V., Cordero, G., Garcés, J., Rodríguez, C., . . Coronel, B. (2019). Tratamiento del dolor postquirúrgico en el servicio de traumatología en un hospital de segundo nivel. Revista Latinoamericana de Hipertensión., 14(2), 194-200. Recuperado el 05 de agosto de 2019, de http://www.revhipertension.com/rlh_2_2019/tratamiento_dolor_postquir\%C3\%BArgico. pdf

Esteve, N., Sansaloni, C., Verd, N., Ribera, H., \& Mora, C. (2017). Nuevos enfoques en el tratamiento del dolor agudo postoperatorio. Rev Soc Esp Dolor, 24(3), 132-139. 


\section{Control del dolor postquirúrgico}

Vol. 3, núm. 2., (2019)

Giannella Lissette Ramírez López; Nancy del Carmen López García; Cinthya Clara Moreira Simbaña; Jenny Marisela Briones Fajardo

Recuperado el 06 de agosto de 2019, de http://scielo.isciii.es/pdf/dolor/v24n3/1134-8046dolor-24-03-00132.pdf

Jiménez, M. B. (2018). Administración intratecal en dosis mínima de morfina para el manejo del dolor postquirúrgico en cesárea. Anestesia en México, 30(3), 38-46. Recuperado el 06 de agosto de 2019, de http://www.scielo.org.mx/pdf/am/v30n3/2448-8771-am-30-03-38.pdf

Mayo Clinic. (20 de noviembre de 2018). mayoclinic.org. Recuperado el 04 de agosto de 2019, de https://www.mayoclinic.org/es-es/pain-medications/art-20046452

NLM. (09 de septiembre de 2017). MedlinePlus. (B. N. Unidos, Editor) Recuperado el 06 de agosto de 2019, de https://medlineplus.gov/spanish/ency/patientinstructions/000406.htm

NLM. (27 de marzo de 2019). MedlinePlus. (B. N. Unidos, Editor) Recuperado el 04 de agosto de 2019, de https://medlineplus.gov/spanish/nondrugpainmanagement.html

Quinde Cobos, P. (2017). DOLOR POSTOPERATORIO: FACTORES DE RIESGO Y ABORDAJE. Medicina Legal de Costa Rica, 34(1), 1-11. Recuperado el 02 de agosto de 2019, de https://www.scielo.sa.cr/pdf/mlcr/v34n1/1409-0015-mlcr-34-01-00254.pdf

Sociedad Española del Dolor. (17 de septiembre de 2017). sedolor.es. Recuperado el 05 de agosto de 2019, de https://www.sedolor.es/60-los-pacientes-intervenidos-quirurgicamente-sufredolor-intenso-postoperatorio-forma-persistente/

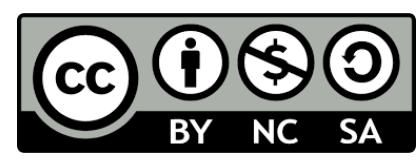

RECONOCIMIENTO-NOCOMERCIAL-COMPARTIRIGUAL

CC BY-NC-SA

ESTA LICENCIA PERMITE A OTROS ENTREMEZCLAR, AJUSTAR Y CONSTRUIR A PARTIR DE SU OBRA CON FINES NO

COMERCIALES, SIEMPRE Y CUANDO LE RECONOZCAN LA AUTORÍA Y SUS NUEVAS CREACIONES ESTÉN BAJO UNA LICENCIA CON LOS MISMOS TÉRMINOS. 